Arthroskopie 2012 $25: 5-6$

DOI 10.1007/s00142-011-0673-9

Online publiziert: 25. Januar 2012

(c) Springer-Verlag 2012

S. Scheffler ${ }^{2} \cdot$ R. Becker ${ }^{1}$

${ }^{1}$ Zentrum für Orthopädie und Unfallchirurgie, Städtisches Klinikum Brandenburg, Brandenburg/Havel

${ }^{2}$ Knie- und Sportorthopädie, Chirurgisch-Orthopädischer Praxisverbund (COPV), Berlin

\title{
Allografts in der Sportorthopädie
}

Lebensdauer des Transplantats erhebliche Unterschiede zwischen den Gewebearten bezüglich des biologischen und mechanischen Verhaltens im Vergleich zum körpereigenen Gewebe gibt. Das differenzierte Wissen über das häufig verzögerte biologische Einheilungsverhalten von Spendergewebe und dessen mögliche Bedeutung für die mechanische Funktion muss Berücksichtigung zum Beispiel bei der Nachbehandlung unserer Patienten finden.

sătzliches Trauma für den Körper und führt häufig zu einer Funktionsstörung ursprünglich nicht geschädigter Strukturen. Andererseits ist man in der Gelenkchirurgie auch mit einer Situation konfrontiert, die eine Limitierung der Rekonstruktion verletzter Strukturen durch fehlendes autologes Material verursacht. Hieraus resultiert die Motivation, alternative Gewebearten nutzen zu können. Neben den immer wieder unternommenen Versuchen, künstlich hergestellte Gewebeanaloga einzuführen, mit größtenteils nur begrenztem klinischem Erfolg, bildet das humane Gewebe von Spendern, so genannte Allografts, eine wichtige Alternative für die rekonstruktive Chirurgie.

Der Vorteil von humanem Spendergewebe liegt in der optimalen Gewebeeigenschaft, die bisher durch keine Analoga erreicht wurde. Allografts werden heute vom Knochen über Sehnen und Bänder bis zum Meniskus und Knorpel in der rekonstruktiven Gelenkchirurgie eingesetzt. Die klinische Sinnhaftigkeit des Nutzens von Spendergewebe bestätigte in den vergangenen Jahrzehnten eine Vielzahl von Studien. Jedoch hat die inzwischen langjährige Erfahrung mit dem klinischen Einsatz von Spendergewebe auch gezeigt, dass es in Abhängigkeit von der

\section{》) Der Vorteil von humanem Spendergewebe liegt in der optimalen Gewebeeigenschaft}

Zusätzlich ergeben sich durch die Notwendigkeit der Entnahme, Lagerung und Transplantation Gefahren von Infektionsübertragungen oder Gewebekontaminationen. Der Umgang mit diesen Risiken unterscheidet sich in Europa erheblich vom angloamerikanischen Raum, wo der Einsatz von Spendergewebe in der arthroskopischen Chirurgie deutlich mehr verbreitet ist. Rechtsgebende Institutionen im deutschsprachigen und europäischen Raum erfordern heute die Erfüllung klarer Richtlinien bei der Prozessierung von Spendergewebe, die jedoch zu einer Bescher Eigenschaften führen können. Das Verständnis des Einflusses der verschiedenen Prozessierungsverfahren auf die Gewebequalität stellt die Grundvoraussetzung dar, um das passende Spendergewebe einschließlich der Verfahren der Aufbereitung zu definieren. Nur so kann eine risikofreie Nutzung gewährleistet werden. einträchtigung mechanischer und biologi-
Aufgrund der rechtlichen Auflagen in Europa darf derzeit nichtprozessiertes Spendergewebe, das in Europa hergestellt wurde, keine Nutzung finden. Bestimmte Eingriffe, wie zum Beispiel die Meniskustransplantation oder der Einsatz osteochondraler Allografts, werden daher in sehr eingeschränkter Anzahl vorgenommen. Jedoch erlaubt die aktuelle europäische Rechtssprechung Ausnahmen, die die Einfuhr und Verwendung auch von solchem Gewebe gestatten. Diese Ausnahmen gilt es zu kennen, und der rechtskonforme Umgang ermöglicht es, Zugang auch zu diesen Geweben zu erhalten. In mancher alternativloser Behandlungssituation unserer Patienten stellen diese Allografts die letzte Option dar.

Während in der Vergangenheit eine unüberschaubare Anzahl lokaler und überregionaler Gewebebanken existierte, haben die erheblichen Auflagen bezüglich des Führens solcher Einrichtungen zu einer deutlichen zahlenmäßigen Reduktion in Europa geführt. Es ist wichtig, auch diese Aspekte zu erwähnen, damit der Anwender nachvollziehen kann, ob das Spendergewebe rechtskonform hergestellt wurde und rechtliche Verantwortlichkeiten klar nachvollziehbar sind.

Dieses Heft gibt Ihnen, liebe Leser, einen Überblick über die Möglichkeiten des Einsatzes von Spendergewebe in der rekonstruktiven arthroskopischen Chirurgie, die speziell unter den besonderen Rahmenbedingungen, die in den deutschsprachigen Ländern existieren, betrachtet werden. Dadurch soll der rechtskonforme, sichere und vor allem klinisch sinnhafte Einsatz von Spendergewebe sichergestellt werden, der häufig das Ende der biologischen Rekonstruktions- 
möglichkeiten von Gelenkpathologien unserer Patienten darstellt.

Ihre

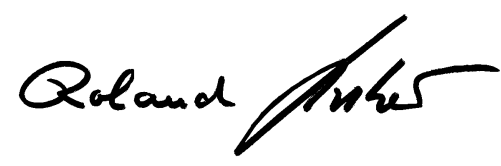

Prof. Dr. R. Becker

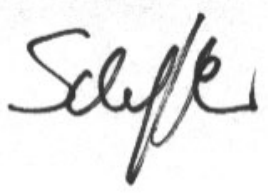

Dr. S. Scheffler

\section{Korrespondenzadresse}

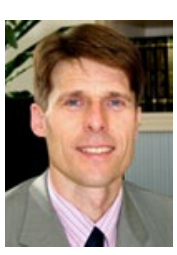

Prof. Dr. R. Becker

Zentrum für Orthopädie und

Unfallchirurgie, Städtisches

Klinikum Brandenburg

Hochstr. 29, 14776

Brandenburg/Havel

r.becker@

klinikum-brandenburg.de

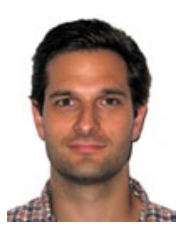

Dr. S. Scheffler

Knie- und Sportorthopädie, Chirurgisch-Orthopädischer Praxisverbund (COPV), Breitenbachplatz 8, 14195 Berlin sven.scheffler@charite.de

\section{EFORT 2012}

Die Europäische Orthopädie und Unfallchirurgie kommt nach Deutschland

Der EFORT Kongress ist der größte internationale Kongress für Orthopäden und Unfallchirurgen in Europa und zieht mittlerweile Teilnehmer aus über 90 Ländern an. Zum zweiten Mal nach 1995 wird nun im nächsten Jahr der Jahreskongress in Deutschland stattfinden: vom 23. bis 25. Mai 2012 lädt EFORT nach Berlin ein und die Teilnehmer erwartet ein hochkarätiges wissenschaftliches Programm. Es sind mehr als 3.900 Abstracts eingereicht worden, eine neue Rekordzahl in der Geschichte der europäischen Gesellschaft.

Aufgrund der UN Dekade für Verkehrssicherheit wird ein Schwerpunktthema die Behandlung von Verletzungen und Verletzungsfolgen sein, ein zweites aufgrund des European Year for Active Aging 2012 die Behandlung der degenerativen Erkrankungen der Haltungs- und Bewegungsorgane.

Dieser Kongress erlaubt in einer einzigartigen Art und Weise die gesamte Breite des Faches zu erfahren, gewürzt durch unterschiedliche länderspezifische Ansichten und internationale Experten der Spitzenklasse.

Beliebte und bewährte Formate wie Symposia und Instructional Lectures (IL) werden durch interaktive Formate wie z.B. Controversial Case Discussions (CCD) und Expert Meet Experts (ExMEx) Sessions ergänzt. Neue Formate, wie etwa Crossfire Sessions, bei denen zwei gegensätzliche Ansätze zur Problemlösung vorgestellt und diskutiert werden, erlauben kontroverse Diskussionen über unterschiedliche Philosophien mit Meinungen aus verschiedenen Ländern. EFORT's Specialty Societies haben Sitzungen zu verschiedenen Schwerpunktthemen vorbereitet, in denen ausgewiesene Experten ihre Erfahrung mitteilen.

Der eintägige Comprehensive Review Course (CRC) richtet sich vorwiegend an jüngere Kollegen und erlaubt einen kompetenten Überblick über das gesamte Fach an einem Tag.

Wir freuen uns darauf mit Ihnen die internationalen Gäste in der deutschen Hauptstadt begrüßen zu dürfen, die für viele ausländische Kollegen eine besondere Anziehungskraft hat. Wir sind überzeugt, dass es auch für die deutschen Orthopäden und Unfallchirurgen eine einmalige Möglichkeit ist, sich in diesem Rahmen fortzubilden und zudem mit internationalen Ansichten auseinandersetzen zu können.

Als EFORTs Kongresspräsidenten und Organisationsteam freuen wir uns, Sie im Mai 2012 in Berlin begrüssen zu dürfen. Sie finden alle weiteren Informationen auf http://www.efort. org/berlin2012/.

Prof. Dieter C. Wirtz und Prof. Karsten E. Dreinhöfer Dreinhöfer mit Florian Gebhardt, Ulrich Stöckle, Karl-Dieter Heller und Klaus-Peter Günther 